\title{
Des cendres au soleil levant
}

C'est au soleil levant que nous avons enterré ses cendres, près de celles de l'Enfant et de l'Ancêtre. Aucun signe ne marque l'endroit où elles sont ensevelies. ${ }^{1}$

Oui, la musique pourrait être ce fond d'âme commun à tous, ò̀ la solitude de chacun est tissée de mille voix. ${ }^{2}$

Bien loin du rap et du slam dont Jean-Claude Pinson affirme qu'ils témoignent aujourd'hui «le plus ouvertement de cette incidence du son de notre époque sur la façon d'articuler et de sonder la langue $»^{3}$, et envisageant les liens que peuvent entretenir musique et poésie, nous nous proposons d'être à l'écoute des résonances entre le rêve que fait le personnage de Diotime à la fin de Diotime et les lions d'Henry Bauchau et Le rêve de Diotime de Pierre Bartholomée. Pour le dire plus justement, nous nous risquons à une rêverie. Du littéraire au musical, deux arts dialoguent sans pour autant lier complètement leur devenir. Ils en appellent tous deux au silence, ils en appellent à l'in-commencement de la voix et entrent dans la fortune du rêve. Lorsque tout s'éteint, lorsque le rêve s'enfuit et que la musique s'arrête, ne restent plus alors que « des cendres au soleil levant ».

Le titre que nous avons choisi vient d'un rapprochement effectué entre deux origines ensevelies. Nous savons depuis Freud que le rêve est la voie royale d'accès aux profondeurs de l'inconscient. Nous savons aussi qu'il est un motif récurrent dans l'œuvre d'Henry Bauchau. Et c'est encore du rêve qu'il s'agit pour la pièce de Pierre Bartholomée. Avec la musique et le chant, il semble que le contenu manifeste du rêve, pour

\footnotetext{
${ }^{1}$ DL97, p. 61.

2 Jean-Michel Maulpoix, La musique inconnue, Paris, José Corti, 2013, p. 33.

${ }^{3}$ Jean-Claude Pinson, Poéthique. Une autothéorie, Seyssel, Champ Vallon, 2013, p. 140.
} 
reprendre la terminologie freudienne, parvienne à faire entendre plus directement son contenu latent, même si celui-ci garde son caractère énigmatique. Mais il faut en passer par le rappel du récit d'Henry Bauchau pour mieux faire le rapprochement entre les cendres et le soleil levant.

Diotime et les lions est ce court récit publié en 1991 qui constituait un épisode du roman Edipe sur la route, mais a finalement été publié à part à la demande de l'éditeur. Dans le roman, Diotime campe un personnage féminin qui occupe la fonction de guérisseur, elle est aussi l'initiatrice d'CEdipe. À ce titre, elle n'est pas sans lien avec la figure de la psychanalyste Blanche Reverchon-Jouve, première psychanalyste de Bauchau. CEdipe, lui, figure le double littéraire de Bauchau. Le récit Diotime et les lions raconte l'enfance de Diotime. Personnage inspiré à la fois de la Diotime de Platon et de la Diotima de Hölderlin, Diotime appartient à une lignée perse, celle de son grand-père paternel Cambyse, «dont les plus lointains ancêtres étaient les lions ${ }^{4}$. La lignée entretient un véritable culte vis-à-vis des lions et c'est naturellement qu'une fois par an a lieu « une guerre rituelle qui dur[e] deux jours et une nuit». En grandissant, Diotime éprouve le désir de participer à cette fête, alléguant qu'il y a déjà eu dans leur clan «des déesses lionnes aussi terribles, aussi puissantes que les lions » et qu'à ce titre elle peut prendre part au combat. Cambyse accepte de changer la tradition du clan : "Nous ferons naître une autre tradition et tu en seras l'initiatrice $»^{5}$, dit-il à sa petite-fille. Ainsi débute l'initiation de Diotime.

Arsès, un des personnages du rêve de Diotime qui nous intéresse ici, est celui qui, venu de Grèce pour proposer à Cambyse la réunification du clan, depuis longtemps séparé, devient l'époux de Diotime. Mais pour prétendre à cette union et devenir chef de clan, Arsès doit au préalable se soumettre à un rite ancestral : tuer un lion. Pour cela, Diotime et son père Kyros l'entraînent en vue du combat et lui apprennent à "penser lion $»^{6}$. Le hasard désigne à Arsès un lion que le clan appelle «le Grand lion», «le Grand Fauve» ou "l'Ancêtre ». Mais ce lion n'est pas seulement l'image de l'ancêtre : «Il [est], vivant dans le même temps que lui, l’image animale, seigneuriale, de Cambyse ». Or, Arsès est désigné

\footnotetext{
${ }^{4}$ DL97, p. 11.

${ }^{5}$ Ibid., pp. 12-13.

${ }^{6}$ Ibid., p. 39.
} 
dans le récit comme «le maître secret des lions»: «S’il comba[t] le Fauve, il finir[a] par le vaincre, et par tuer cette incarnation mystérieuse de Cambyse et sans doute Cambyse lui-même ${ }^{7}$. Arsès et Diotime se rendent donc auprès d'un Vieillard, un sage, auprès de qui ils espèrent trouver une solution à ce problème cornélien. Là, intervient, une nuit, le rêve de Diotime :

Cette nuit-là j’ai rêvé de lions et d'un combat dont Arsès était sur le point d'être vainqueur. À ce moment une vague énorme s'est élevée dans la mer. Elle s'avançait vers nous dans le grondement du tonnerre. Elle était très haute, très bleue et parsemée de soleils rouges. Étendue sur la rive pour mieux voir la victoire d'Arsès, $j$ 'ai voulu me lever, m'enfuir alors qu'il était évident que la vague qui se ruait vers nous allait tout engloutir. Quelque chose a pensé : Cesse de t'agiter. Reste où tu es. Arsès a dû penser la même chose car il n'a pas bougé non plus. J'ai alors été transportée de joie par la beauté de la vague. De la couleur du soleil levant, elle s'était arrêtée au milieu de la mer. Elle était suspendue au-dessus de nous, portant à son sommet sa terrifiante chevelure d'écume. Elle brillait, elle éclairait, elle nous réchauffait sans cesser un instant d'être très menaçante. Je ressentais tout le bonheur qu'il y avait à être, à être là et je me suis éveillée. ${ }^{8}$

Diotime raconte son rêve au Vieillard, figure de Lao Tseu, qui l'initie à la discipline du Tao, et la jeune fille apprend à accepter la vie comme elle va. À la fin de Diotime et les lions, Cambyse, le grand-père, meurt et ses cendres sont enterrées au soleil levant, "aucun signe ne marque l'endroit » et le lieu « est demeuré sacré » ${ }^{9}$. Pour le dire autrement, à la fin du récit, quelque chose d'un mystère des origines disparait, s'évanouit, est enfoui dans les profondeurs de l'histoire.

La vague est l'élément primordial évoqué dans le rêve. Elle symbolise l'ennemi à combattre. Elle est personnifiée si nous considérons la formule «la terrifiante chevelure d'écume», qui fait penser à la terrifiante chevelure de serpents des Gorgones. Dans le rêve, Diotime et Arsès ne bougent pas face à son envergure impressionnante. Nous pourrions dire qu'ils sont médusés. Mais si habituellement, l'une des Gorgones, Méduse, pétrifie d'horreur, la vague, elle, transporte Diotime de joie. Il s'agirait ici

\footnotetext{
${ }^{7}$ Ibid., pp. 42-43.

${ }^{8}$ Ibid., pp. 51-52.

${ }_{9}^{9}$ DL97, p. 61.
} 
d'une vague conjuguant à la fois le caractère terrifiant de Méduse et celui, sidérant, des Néréides, petites filles d'Océan, qui passent pour être d'une grande beauté.

La vague du rêve " est arrêtée au milieu de la mer ». Quelque chose se fige ainsi, en suspens, qui se présente dans une tension extrême. Le rêve de Diotime reste dans une irrésolution, ce que tendent à confirmer les phrases «[e]lle brillait, elle éclairait, elle nous réchauffait sans cesser un instant d'être très menaçante. Je ressentais tout le bonheur qu'il y avait à être, à être là et je me suis éveillée » - leur amplitude ternaire, leur cadence mineure et la gradation que nous pouvons y lire portent vers cette sensation de mise en tension. Tenant compte de la symbolique et du texte lui-même, cette sorte d'arrêt sur image ne permet pas d'appréhender quelle sera l'issue du combat que s'apprête à livrer Arsès. Cette indécision est portée par des expressions qui indiquent qu'une action est sur le point de survenir: "sur le point d'être vainqueur ", "allait tout engloutir», "arrêtée au milieu de la mer», "suspendue au-dessus de nous ». Toutes participent de cette mise en tension extrême, sans issue connue pour le combat puisque Diotime s'éveille et le rêve retourne dans les profondeurs de l'inconscient.

L'œuvre musicale de Pierre Bartholomée, Le Rêve de Diotime, visite en quelque sorte cette part de mystère enfoui dans les profondeurs de l'inconscient. De la même façon que pour l'ancêtre, au soleil levant, ne restent que des cendres (ainsi le dit la fin du récit), au réveil de Diotime, il ne reste que les cendres du rêve. Ce rêve marque la transformation de Diotime en une sorte de sage. Elle sort du désir pour entrer dans l'équanimité.

Il ne s'agit pas pour le compositeur de rendre, par la musique et le chant, une équivalence du mouvement de la phrase bauchalienne, de son contenu ou de son émotion. Pierre Bartholomée travaille «à partir d'éléments extrêmement restreints pour en tirer le plus de conséquences et de substance, dans une écriture la plupart du temps vocale et instrumentale qui explore les régions extrêmes ${ }^{10}$. Il fait résonner le son pour l'apparition d'un être de lumière dont notre esprit cherche sans cesse les

10 Pierre Bartholomée, entretien avec Isabelle Françaix, propos recueillis le 11 décembre 2009 à Ottignies, disponible en ligne, à l'adresse : www.musiquesnouvelles.com/fr/ Entretiens/Pierre_Bar tholomee (page consultée le 15 décembre 2014). 
contours sans jamais pouvoir parvenir à l'atteindre. En ce sens, si la tension musicale est permanente dans un envahissement de l'espace du rêve et selon une sorte d'appel, elle est comparable au soleil levant qui darde ses rayons et laisse apparaitre le monde, ou plutôt appelle le monde à la lumière.

Entre l'ouvert du langage et l'espace musical, entre une écriture défaillante, qui ne peut restituer que les bribes du rêve, lui-même déjà lacunaire par nature, et une musique de la tension, qui use d'instruments et d'une voix de soprano, celle de Valentina Valente, un espace de résonance se crée. Il semble qu'au sein de ce qui les gouverne toutes deux, écriture et musique soient animées de mouvements contraires: abîme du rêve pour la première et élévation du son ou érection du désir $\mathrm{du}$ rêve pour la seconde. La transposition musicale de Pierre Bartholomée nourrit alors davantage notre imaginaire, ou enrichit notre lecture du récit d'Henry Bauchau.

Selon Pierre Jean Jouve, «la Musique est liée à la circulation du sang, à la formation de la conscience, à l'inconscient même. Elle est directement entée sur la force vitale ${ }^{11}$. L'œuvre musicale de Pierre Bartholomée est également entée sur cette force vitale, sur «un instinct religieusement écouté, au milieu du silence imposé à tout le reste $»^{12}$, comme l'écrit Proust de l'œuvre littéraire dans Le Temps retrouvé. Pour avoir une idée - même assez vague - de cette force vitale, il faut écouter Le Rêve de Diotime dans le silence de façon à ne plus entendre du son que cette tension extrême que la composition de l'opus agrémente du rêve ou du fantasme de l'auditeur. Les aigus de la voix de soprane sont cette tension de la vague qui s'est élevée et reste ainsi, immobile, comme les personnages sont extatiques. Ce point, proche du point de rupture, n'est pas exactement le pic du rêve, car celui-ci, exprimé par le chant, connaît des variations, comme la nuit de l'homme n'est pas linéaire mais habitée de variations. Mais il porte le corps dans un état d'inquiétude.

Il est possible de retrouver dans la musique et dans le chant les synesthésies et les correspondances baudelairiennes, et la musique dou-

11 Pierre Jean Jouve, "L'oiseau magique », dans En Miroir, dans Euvres II, Paris, Mercure de France, 1987, p. 1179.

12 Marcel Proust, Le Temps retrouvé, dans À la recherche du temps perdu, Paris, Gallimard, «Quarto », 1999, p. 2283. 
blée par le chant donne à voir, comme Éluard le pense de son poème. Assurément, ils prolongent l'épisode, ou l'élargissent, ouvrent davantage à l'espace de l'inconscient et entretiennent l'énigme du rêve. Ils dessinent une sorte d'arabesque au sens où l'entend Jean-Yves Tadié, c'est-à-dire une "extase prolongée en rêverie $»^{13}$. La musique et le chant de Pierre Bartholomée proposent une arabesque mélodique qui traduit une émotion. Il faut entendre peut-être également cette arabesque au sens où Mallarmé la comprend dans La Musique et les Lettres, c'est-à-dire comme ce qui relie «l'ambiguïté de quelques figures belles, aux intersections ", et possède «de vertigineuses sautes» et «d'anxieux accords». Elle est structurée « de ces motifs qui composent une logique, avec nos fibres $»^{14}$. Chez Pierre Bartholomée, nous pouvons entendre dans l'arabesque une expression musicale de la variation et de la tension, habitée parfois par le silence ou qui tend à s'amuïr dans le silence, mais qui a une incidence sur le corps de l'auditeur, sur son rythme, sa respiration, sa marche. L'arabesque assure la continuité du rêve, sa survivance, après même qu'il s'est achevé. Il s'agit d'un rêve musical comme flux qui roule le rêve dans la caillasse du rêve. Il existe une sorte de perte de repères que le son ménage dans une tension continue. Sans doute cette sorte de vibrato du violon exprime-t-elle la disharmonie pour l'harmonie. Comme dans la danse, jamais l'arabesque ne s'arrête ni se clôt sur elle-même, elle est un prolongement continu. D'où ce goût de cendres au soleil levant quand le rêve s'effondre et que Diotime s'éveille, dû à la fois à l'espérance qui se dégage du rêve, à son empêchement à se conclure puisque le rêve de Diotime s'interrompt sur la levée de cette magnifique vague, et au sentiment terrifiant du danger imminent.

Nous remarquons que Le Rêve de Diotime possède un élément supplémentaire par rapport au rêve du récit de Bauchau. Le chant débute par trois phrases nominales qui sont comme une façon de mettre en scène le combat contre les lions: "les rugissements des fauves », "les hennissements des chevaux », «le tintement des armes ». La phrase mélodique, pour chacun de ces éléments, va crescendo des graves aux aigus et l'épisode d'ouverture qu'elles constituent se referme sur un mouvement inverse qui reprend «les rugissements des fauves» des aigus vers le

\footnotetext{
13 Jean-Yves Tadié, Le songe musical, Paris, Gallimard, «L'un et l'autre », 2008, p. 167.

14 Stéphane Mallarmé, La Musique et les Lettres, dans Igitur, Divagations, Un coup de dés, Paris, Gallimard, « Poésie », 2001, p. 357.
} 
grave. Le tout se clôt sur l'entrechoc des cymbales puis par la ponctuation de la grosse caisse. Enfin, le silence s'installe pour un instant avant que le rêve de la vague ne survienne. Certes, nous pouvons considérer que le compositeur a choisi cette introduction comme mise en scène de la première phrase du rêve : "Cette nuit-là j’ai rêvé de lions et d'un combat». Mais il semble que Pierre Bartholomée ait réuni deux rêves de Diotime. Le premier se situe, dans le récit de Bauchau, au début, au moment où la jeune fille décide d'obéir à sa mère et renonce, pour un temps, au combat contre les lions. Allant ainsi contre sa nature profonde, c'est là qu'elle rêve et que ce rêve s'enfonce ensuite dans le délire. C'est lors de ce premier rêve qu'elle frappe Cambyse, assimilé au lion, dans une sorte de combat. Les trois phrases ajoutées par Pierre Bartholomée avant le rêve de la vague proprement dit sont peut-être présentes pour rappeler que le personnage ne se réalise que dans ce combat contre les lions, que c'est là sa vérité profonde. Le texte de Bauchau ne dit-il pas que c'est dans le combat contre les lions que Diotime éprouve «élan, ouverture et libération $»^{15}$.

Puis, alternant moments d'exaltation et de retenue, que la musique et le chant soutiennent de la puissance instrumentale et de la hauteur de la voix, quelque chose d'une disharmonie ou d'une dislocation conjugue ses ruptures pour assurer la continuité du rêve. Profusion, désordre, souffle, tension extrême des cordes et de la voix, élévation, accalmie, inquiétante étrangeté, récurrence du mouvement musical, amplification, ponctuation du silence qui joue le rôle d'une sorte de reflux de l'idée comme si celleci prenait alors le temps de l'imprégnation des couches profondes de l'être : tout dans l'œuvre de Pierre Bartholomée dit l'emportement de Diotime dans le rêve. La voix insistante, ou traînante, sur l'adjectif verbal «suspendue » qui détermine la vague, et qui se prolonge dans un écho, tend à dire l'emportement du personnage dans le flux onirique, son enlèvement.

Dans ce rêve de Diotime, tout semble s'étirer dans le temps. Cette impression est peut-être due aux imparfaits duratifs de la fin du texte de Bauchau. Mais c'est aussi, du côté de l'œuvre musicale, d'une part, en vertu de la symbolique de la vague qui représente le temps dans l'esthétique orientale, d'autre part, en vertu des nombreux silences qui font le

${ }^{15}$ DL97, p. 15. 
lien entre deux épisodes sonores, que le mouvement semble s'étirer à l'infini. S'installer dans le rêve, c'est s'installer dans le temps du rêve, dans ce temps incommensurable qui prolonge le combat rituel sans jamais l'accomplir, sans jamais opter pour une victoire. La vague qui se dresse exprime le danger imminent, le moment décisif d'avant l'issue du combat. Mais interrompre le rêve avant l'issue fatale renvoie la destinée de Cambyse dans les profondeurs de l'inconscient, dans les cendres au soleil levant, dans les cendres du rêve au réveil de Diotime.

Pierre Bartholomée a changé un élément textuel par rapport au texte de Bauchau. Si ce dernier écrit « quelque chose a pensé : Cesse de t’agiter. Reste où tu es ", lorsque Diotime voit cette immense vague s'élever face à elle, le compositeur a choisi de transformer la phrase en «j’ai pensé ». Là où Bauchau note l'inaccessibilité de la source du rêve et de la survenue du langage, souligne l'in-commencement de la voix, comme dans la création, Pierre Bartholomée réassocie la parole du rêve à la subjectivité du personnage comme appropriation de l'événement.

Ajoutant des silences, nous l'avons dit, qui étirent le temps du rêve, le compositeur fait entrer le personnage dans le mouvement de la contemplation. Ainsi en est-il notamment d'un silence particulier après l'expression «la beauté de la vague ». Ce ravissement de Diotime sous l'enchantement, ou plutôt la sidération de la vague est l'équivalent probable de celui du poète par la beauté. Là, comme le note Gérard Titus-Carmel pour son poème, «l'ombre [du] corps viendrait exactement coïncider avec le rêve qui le porte $»^{16}$.

Si nous convoquons la beauté, c'est que la poésie, comme la beauté, «se tient dans l'entre, dans ce qui jaillit de plus haut quand, un bref instant, on réussit - ou croit réussir - à se frayer un passage dans l'opacité des mots. Une lumière, alors, indique où l'impossible foudroie ${ }^{17}$. Ce que la musique et le chant possèdent de beauté par rapport au texte de Bauchau, c'est leur caractère déréalisant, devenant en ce sens plus proches du rêve. La beauté devient cette abstraction qu'est la vague. Musique et chant portent l'essence même du rêve car si le langage est la matière première du rêve bauchalien, c'est le son qui porte le rêve chez

16 Gérard Titus-Carmel, Le buitième pli on Le travail de beauté, Paris, Galilée, «Écritures/Figures », 2013, p. 28.

${ }^{17}$ Ibid., p. 141. 
Pierre Bartholomée, comme un originel du signe. Sa subtile puissance musicale est sa merveille.

Nous faisons l'hypothèse que cette beauté de la vague entretient des liens avec le sacré si nous en revenons à l'étymologie du mot (sacer). En effet, sacer dénote à la fois « la présence d'un signe surhumain vénérable (proche d'angustus) et une mise à l'écart par une souillure qui suscite l'effroi $»^{18}$. Cette dialectique du sacré est celle de l'effroi et de la vénération, de la terreur et de la confiance, du tremendum et du fascinans. Rudolf Otto "rattache le sacré à une structure émotionnelle a priori, le numinosum, qui se rapporte à l'impression qu'a la conscience d'être conditionnée par une force indépendante de sa volonté, le Tout Autre $»^{19}$. Or ce Tout Autre chez Bauchau s'impose avec toute la violence de la révélation, et ce n'est certainement pas un hasard si la seule définition du sacré que l'on trouve dans son œuvre, et qu'il emprunte à René Girard, soit justement celle qui l'unit à la violence :

René Girard: "Le sacré, c'est tout ce qui maittrise l'homme d'autant plus sûrement que l'homme se croit plus capable de le maittriser. C'est donc entre autres choses mais secondairement, les tempêtes, les incendies de forêt, les épidémies qui terrassent une population. Mais c'est aussi de façon bien plus cachée, la violence des hommes eux-mêmes, la violence posée comme extérieure à l'homme et confondue, désormais, à toutes les autres forces qui pèsent sur l'homme du dehors. C'est la violence qui constitue le cœur véritable et l'âme secrète du sacré » (La Violence et le Sacré). ${ }^{20}$

Si René Girard fait allusion à la violence en ce que les récits fondateurs des religions évoquent presque toujours un meurtre primordial qui devient l'événement sacré à réactualiser lors de cérémonies, il ne s'agit pas exactement de meurtre dans le rêve de Bauchau, mais plutôt

\footnotetext{
18 Jean-Jacques Wunenburger, Le sacré, Paris, Presses universitaires de France, «Que saisje?», 2001, p. 4.

${ }^{19}$ Ibid., p. 9. «Le numineux sert de matrice à des significations aussi variées que celle de transcendant, d'infini, d'absolu, de surnaturel, en un mot de divin. Le divin n'est donc pas d'abord posé comme Idée, mais se trouve réfléchi à travers un émoi sensitivointellectuel, qui n'en retient qu'un reflet indirect, qu'une sorte de trace ou d'écho.» (Ibid., p. 12.)

${ }^{20}$ PBG, 19 septembre 2001, p. 388. «La violence se trouve captée par le sacré, dont elle traduit précisément la puissance numineuse. » (Jean-Jacques Wunenburger, Le sacré, op. cit., p. 58.)
} 
de rêve prémonitoire qui ouvre sur une violence sacrée sous-jacente, ou non formulée. La fin de Diotime et les lions ne dit-elle pas ce lieu sacré de la violence, en réactualisant le rêve de Diotime, lorsqu'est évoqué le lieu où sont enterrées les cendres de Cambyse: "Ce lieu, avec les rochers et l'espace brûlant qui l'entourent, est demeuré sacré. Si des lions et des hommes s'y rencontrent, aucun n'attaque et nul ne fuit $»^{21}$.

Si, parfois, des phrases musicales peuvent hanter jusqu'à l'obsession, ici, avec l'opus de Pierre Bartholomée, c'est l'obsession qui hante jusqu'à la phrase musicale. Du rêve de la vague au vague du rêve comme indécision de l'image qui se donne à voir, "Énigme, ce qui nait d'un jaillissement pur! Et par/ Le chant lui-même à peine dévoilée $»^{22}$, pourrionsnous dire en empruntant à Hölderlin. Si le rêve, le langage du rêve, ou plutôt ses langages, sont à la source de ces deux œuvres, littéraire et musicale, peut-être sommes-nous en présence de deux arts qui creusent leur devenir comme une remontée à leur source. Ils guettent non pas ce qui les rapproche mais ce qui constitue le fondement même du rêve, c'est-à-dire une impossibilité structurelle à identifier ce qui les fait naître à eux-mêmes.

Bartholomée réussit à porter ailleurs le rêve bauchalien. Il déplace l'enjeu. Quand l'imaginaire, le fantasme, l'inconscient du lecteur, sont animés dans le texte de Bauchau, c'est le corps dans son entier qui est transporté dans le domaine du rêve avec la composition de Pierre Bartholomée. La musique et le chant apportent une résonance supplémentaire. Le corps entre en vibration, comme lorsque nous rêvons, suivant le rythme respiratoire de l'opus. L'auditeur en sort en ayant éprouvé une perte et un renouveau; il a été traversé par une onde de choc dont il se souviendra longtemps.

Régis LEFORT

Aix Marseille Université

${ }^{21}$ DL97, p. 61.

22 Friedrich Hölderlin, «Le Rhin», dans Hymnes, dans CEuvres, Paris, Gallimard, «Bibliothèque de la Pléiade », 1995, p. 850. 